\title{
Low-Voltage Current-Mode WTA/LTA Circuit
}

\author{
Behzad Ghanavati", Enayatollah Taghavi Moghaddam \\ Department of Electrical Engineering, Mahshahr Branch, Islamic Azad University, Mahshahr, Iran \\ *Corresponding Author: B.ghanavati@mhriau.ac.ir
}

Copyright @ 2014 Horizon Research Publishing All rights reserved.

\begin{abstract}
A low-voltage CMOS current-mode Winner take all and looser take all circuits are designed in $0.35 \mu \mathrm{m}$ standard CMOS technology.Simulation results using HSPICE that verify the functionality of circuit with $1.5 \mathrm{~V}$ supply are presented. The circuit can find application in the implementation of Fuzzy and Neural Network circuits.
\end{abstract}

Keywords Analog processing circuit, WTA, LTA, Low Voltage

\section{Introduction}

Winner-Take-All (WTA) and Looser-Take-All (LTA) circuits are the analog important and major function building blocks. The function of WTA and LTA circuits identify the largest and the smallest input variable, respectively, and restrains the remaining ones. WTA and LTA are widely used in hardware implementation of fuzzy logic systems, nonlinear filters and self-organizing neural networks, vector quantization, Hamming network, competitive learning, etc.

WTA and LTA can be extensively categorized as the current mode and voltage mode structure. Using current mode analog circuit design has received wide attention due to the supply voltage scaling down and their potential of lower power consumption [1]-[3]. In addition existing voltage mode structures are larger than current mode structures in hardware and area.

In this paper we proposed a novel current mode WTA and LTA. There are several structures of current mode WTA and LTA circuit proposed on the literature [4]-[6]. Our proposed circuits can work under low voltage $(1.5 \mathrm{~V})$ that is lower in comparison with previous works.

Section 2 presents the proposed WTA circuit architecture and describes it operation. Section 3 presents the proposed LTA circuit architecture. Simulation results are presented in section 4. Conclusion is presented in section 5 .

\section{WTA Circuit Description}

The function of the WTA is to accept input signals, compare their values and produce a high digital output value (logic 'one') corresponding to the largest input, while all other digital outputs are set to low output value (logic 'zero') [7].

Figure 1 shows the block diagram of the WTA circuit. The circuit includes a 2-input current maximum selector [8] with a voltage inverter.

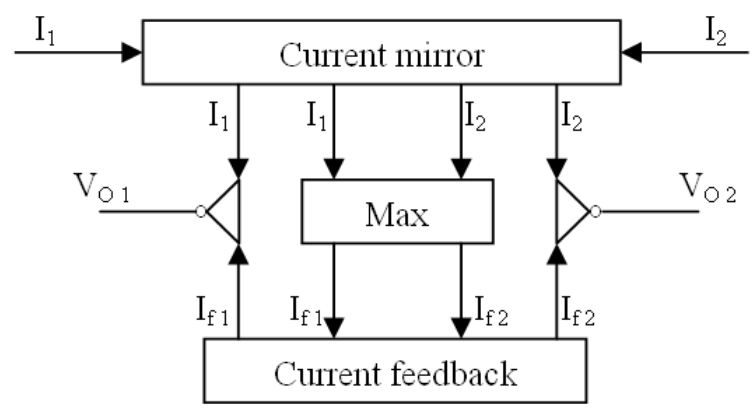

Figure 1. Winner Take All circuit

\subsection{The Current Max Selector}

The 2-input current maximum selector is shown in Figure 2. The proposed current max selector has 2 input branches and each branch consists of an FVF [14], formed by voltage follower Mai, and current sensing transistor Mci.

Transistor Mai in an FVF performs as an improved voltage follower and the Gate-Source voltage drop of this transistor is constant (neglecting second-order effect) and independent of the load

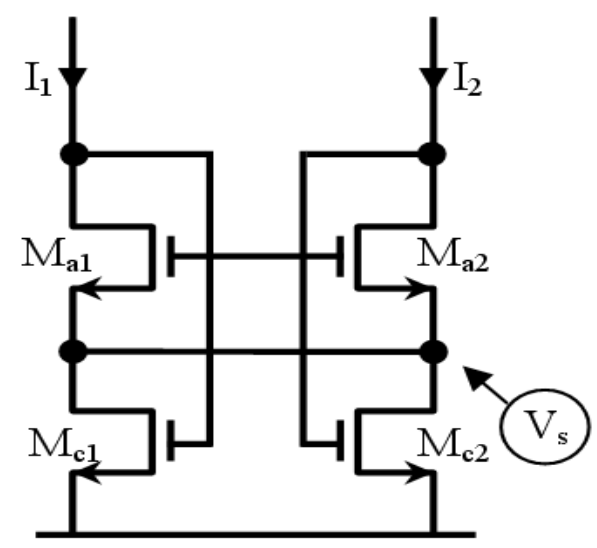

Figure2. 2-input current max circuit 
Transistor Mci operates as a current sensing device. It can sink large current by keeping its Drain voltage approximately constant. Moreover, the existing impedance at the Source of transistor Mai is very low due to the feedback loop.

The principle of operation of the circuit is as follow. The voltage at node "VS" follows the maximum of input currents I1, I2, with a DC level shift VGSn where $\mathrm{n}$ denotes the maximum current.

In this condition the transistor (Ma1 or Ma2) which carrying the minimum current, have the greater Gate-Source voltage than the value that should have to operate in saturation mode, at this condition this transistor operates in triode mode with Drain-Source voltage value close to zero, thus the current sensing transistor is turning off in this branch and minimum and maximum currents passed through current sensing transistor of winning branch due to properties of FVF cell.

\subsection{The Overall Structure of WTA Circuit}

The circuit of the 2-input WTA is shown in Figure 3. The currents (I1, I2) are the inputs of the circuit.

Each current is mirrored into current max selector, as well as, into the feedback circuit due to PMOS current mirror M12, M22.

Thus the input current of each voltage inverter is:

$$
\begin{aligned}
I_{i 1} & =I_{1}-I_{f 1} \\
I_{i 2} & =I_{2}-I_{f 2}
\end{aligned}
$$

We assume at the steady state, the current I1 is the largest input current $\mathrm{I} 1=\max (\mathrm{I} 1, \mathrm{I} 2)$

So

$$
\begin{aligned}
I_{f 1} & =I_{1}+I_{2} \\
I_{f 2} & =0
\end{aligned}
$$

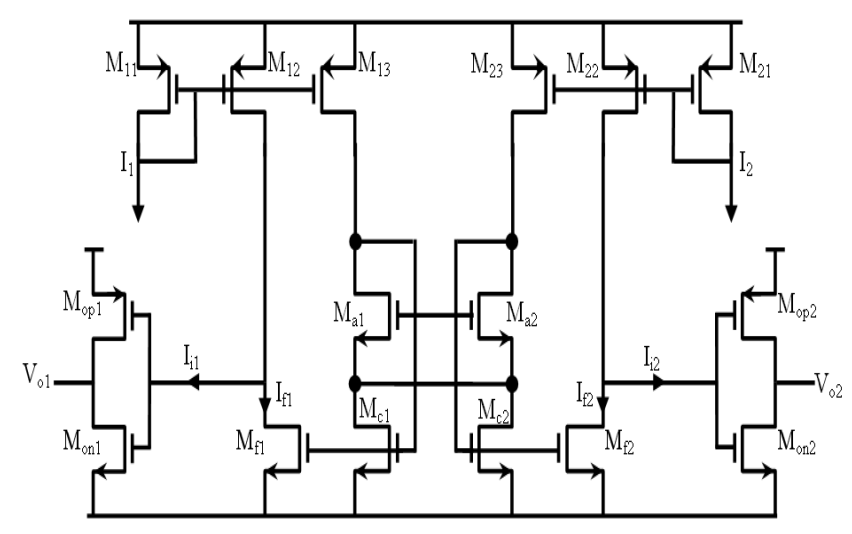

Figure 3. 2-input WTA circuit

From the "Eq. (1)" - "Eq. (4)" the input current of each voltage inverter is:

$$
I_{i 1}=I_{1}-\left(I_{1}+I_{2}\right)=-I_{2}
$$

$$
I_{i 2}=I_{2}-0=I_{2}
$$

This means that only one input current of the voltage inverter corresponding to minimum current is positive and all the other currents are negative. Thus the digital voltage outputs of the circuit will be at logic.

$$
\left\{\begin{array}{c}
V_{O 1}={ }^{\prime} \text { one' } \\
V_{O 2}={ }^{\prime} \text { zero' }^{\prime}
\end{array}\right.
$$

\section{LTA Circuit Description}

A LTA-Min circuit can be formed as shown in Fig.4. The basic structure of the proposed circuit consists of two branches. Each of these branches consists of a voltage follower transistor $\left(\mathrm{MA}_{\mathrm{i}}\right)$ and a current sensing transistor $\left(\mathrm{MC}_{\mathrm{i}}\right)$. The gate of the $\mathrm{MA}_{\mathrm{i}} \mathrm{s}$ is connected to a fixed voltage $\mathrm{V}_{\mathrm{b}}$.

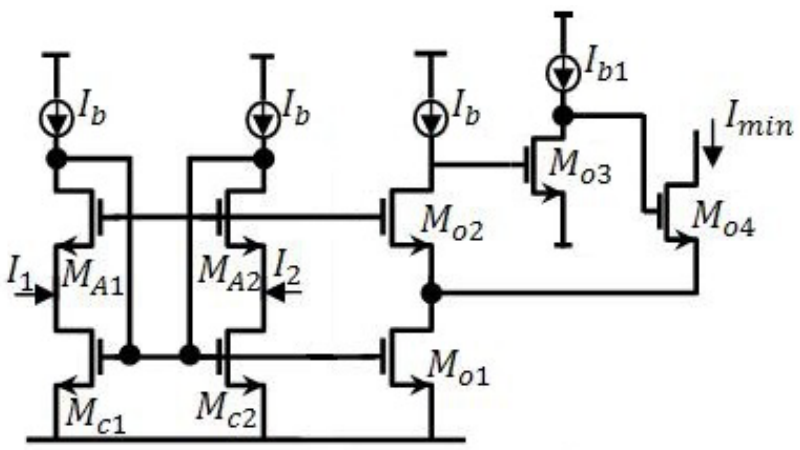

Figure 4. The prposed Minimum current selector

The gate-to-source voltages of $\mathrm{MC}_{\mathrm{i}} \mathrm{s}$ are equal and proportional to minimum currents $I_{1}$ and $I_{2}$ so that these transistors have the different drain-to-source voltages. In hence, voltage follower transistors of all of the branches will be turning off, except one, which is in the looser branch. This voltage follower transistor has the minimum current that transmits it to the output by using high quality and appropriate current mirror $\left(\mathrm{MO}_{1}\right.$ and $\left.\mathrm{MO}_{4}\right)$.

Transistor $\mathrm{M}_{\mathrm{O} 2}$, current source $\mathrm{I}_{\mathrm{b}}$ and $\mathrm{V}_{\mathrm{b}}$ forces the Drain-to-source voltage of transistor $\mathrm{M}_{\mathrm{O} 1}$ to be a constant value to $\mathrm{M}_{\mathrm{ci}}$ which is in the looser branch and carry the minimum current. To have the high output impedance, the output cascade transistor $\mathrm{M}_{04}$ is driven by the drain of transistor $\mathrm{M}_{03}$. As the polarity in the drain of transistor $\mathrm{M}_{02}$ is reversed, an inverting stage is required to drive the gates of transistor $\mathrm{M}_{\mathrm{o4}}$.This Inverting stage provides additional gain-boosting, which increases the output impedance. The inverter amplifier has been implemented by means of transistor $\mathrm{M}_{\mathrm{o} 3}$ and biasing current $\mathrm{I}_{\mathrm{b} 1}$.

The minimum supply voltage is limited by the path formed by current source $I_{b}, M_{b}$ and $M_{C}$, so the minimum supply voltage is expressed as

$$
V_{D D}^{\min }=V_{G S b}+V_{D S C, s a t}+V_{D S b, s a t}
$$

Where $V_{G S B}$ is the gate-to-source voltage of transistor $\mathrm{M}_{\mathrm{b}}$, 
$V_{D S C, s a t}$ is the minimum operating voltage for the current source $\mathrm{I}_{\mathrm{b}}$ and $V_{D S C \text {,sat }}$ is the minimum drain-to-source voltage of transistor $\mathrm{M}_{\mathrm{c}}$ that cause to operate it in saturation region and can be small as $0.15 \mathrm{~V}$ in $0.35 \mu \mathrm{m}$ CMOS technology, $\mathrm{V}_{\mathrm{tn}}=0.6 \mathrm{~V}$. so, the minimum supply voltage is given by

$$
V_{D D}^{\min }=V_{t n}+V_{\text {DSsat }}=0.7+(3 \times 0.115)=1.15
$$

In this paper corresponding to achieved results, we selected $\mathrm{V}_{\mathrm{DD}}=1.5 \mathrm{~V}$ in order to have an appreciable voltage swing.

\section{Simulation Result}

The proposed WTA and LTA were laid out in standard $0.35 \mu \mathrm{m}$ CMOS technology. Post layout simulations from extracted circuit were performed for a $1.5 \mathrm{~V}$ supply using HSPICE and level 49 parameters (BSIM3V3)

Transient simulation result for the proposed MAX circuit is shown in Fig. 5 where the output is indicated with solid lines.

Fig. 6 shows the output transient response of the WTA circuit for two different currents.

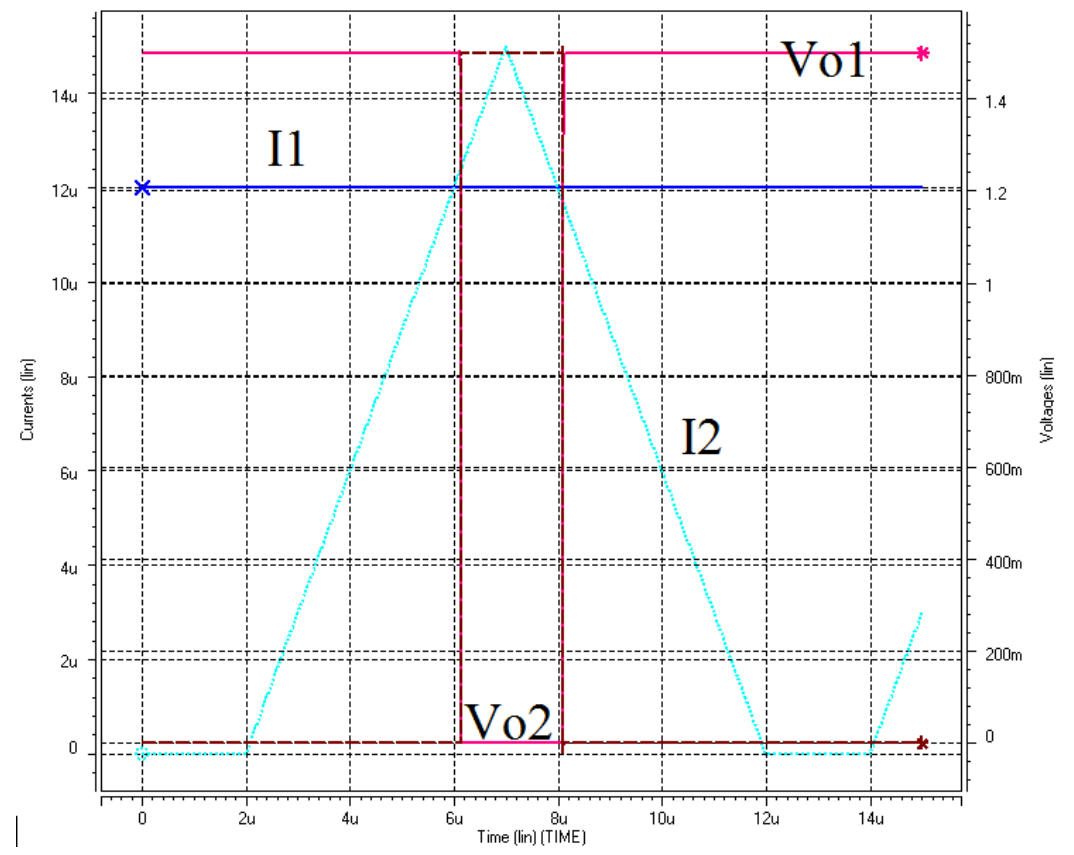

Figure 5. Transient simulation result for the proposed MAX circuit

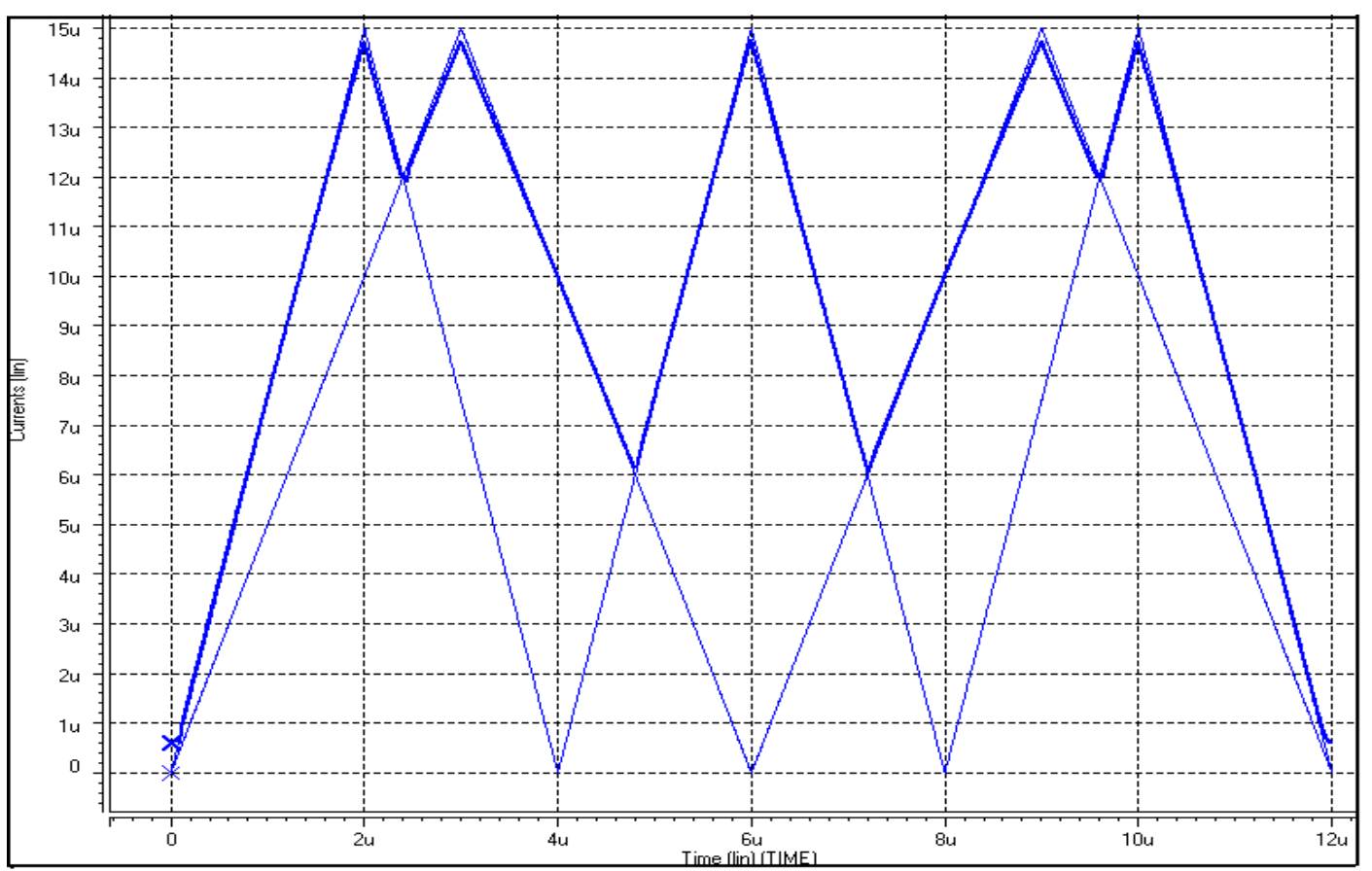

Figure 6. Transient simulation result for the proposed WTA circuit 
Fig.7 shows the transient simulation result for the proposed Minimum current selector in $500 \mathrm{KHz}$. Fig. 8 shows the output of the proposed circuit in $50 \mathrm{MHz}$.

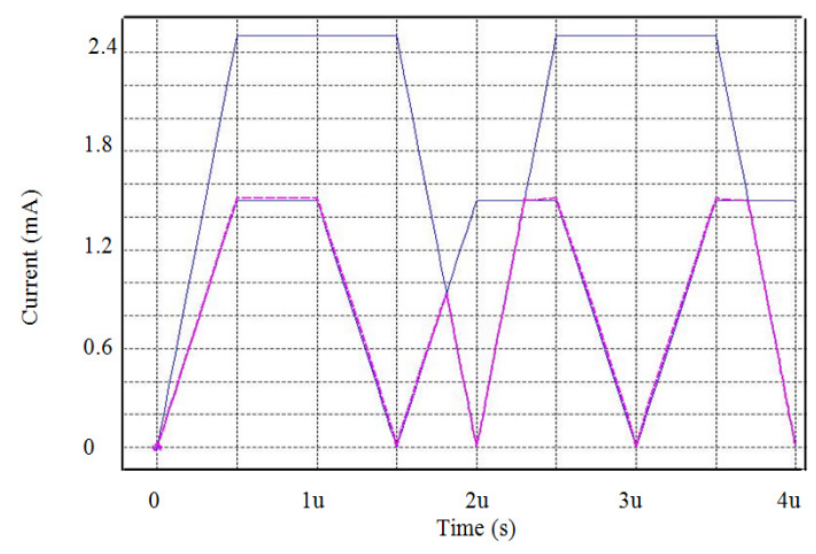

Figure 7. Min circuit operation in $500 \mathrm{KHz}$

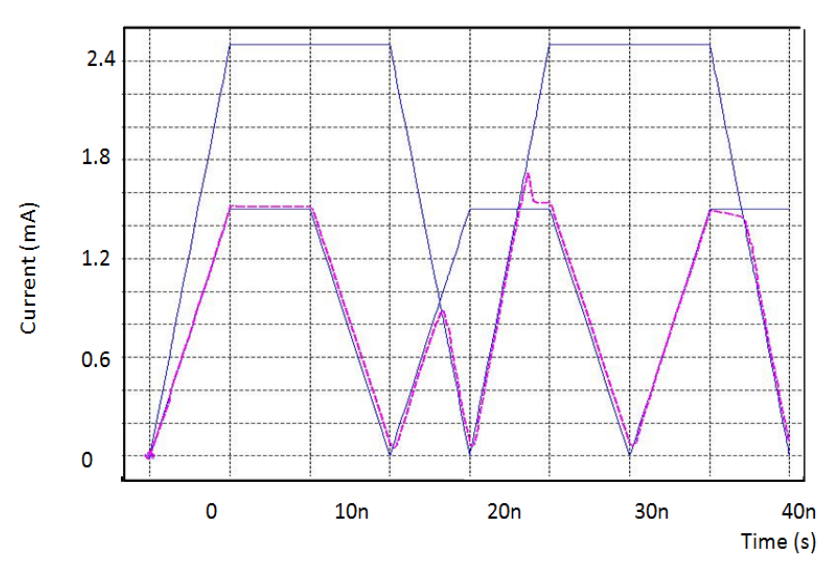

Figure 8. Min circuit operation in $50 \mathrm{MHz}$

\section{Conclusions}

A new, Low-voltage current mode multi input WTA and LTA circuits are described. The proposed circuits work with a single $1.5 \mathrm{~V}$ power supply which makes them suitable for low-voltage portable application.

\section{REFERENCES}

[1] A. Fish, V. Milrud, O. Yadid-Pecht, "High-speed and high-precisioncurrent winner-take-all circuit", Circuits and Systems II: Express Briefs, IEEE Transactions on. Vol. 52, No. 3, 2005, pp. 131-135.

[2] J, Ramirez-Angulo, G. Ducoudray-Acevedo, R. G. Carvajal, A.Lopez-Martin, "Low-voltage high-performance voltage-mode andcurrent-mode WTA circuits based on flipped voltage followers",Circuits andSystems II: Express Briefs, IEEE Transactions on. Vol.52 , No. 7, 2005, pp. 420-423.
[3] Y. Fang, M.A. Cohen, T.G. Kincaid, "Dynamic Analysis of aGeneral Class of Winner-Take-All Competitive Neural Networks",Neural Networks, IEEE Transactions on, Vol. 21, No. 5, 2010, pp.771-783.

[4] Asloni, M., Khoei, A., and Hadidi, K.: 'Design of analog current-modelooser-take-all circuit', IEICE Trans. Electron., 2006, E89-C, (6),pp. 819-822

[5] Huang, C.Y.,Wang, C.J., and Liu, B.D.: 'Modular current-mode multipleinput minimum circuit for fuzzy logic controllers', Electron. Lett., 1996,32, (12), pp. 1067-1069

[6] Dualibe, C., Jespers, P., and Verleysen, M.: 'Embedded fuzzy control forautomatic channel equalization after digital transmissions'. Proc. IEEEInt. Symp. on Circuits and Systems, (ISCAS), Sydney, Australia, 2001,Vol. 3, pp. 173-17

[7] A. Fish, V. Milrud, O. Yadid-Pecht, "High-speed and high-precisioncurrent winner-take-all circuit", Circuits and Systems II: Express Briefs, IEEE Transactions on. Vol. 52, No. 3, 2005, pp. 131-135.

[8] J, Ramirez-Angulo, G. Ducoudray-Acevedo, R. G. Carvajal, A.Lopez-Martin, "Low-voltage high-performance voltage-mode andcurrent-mode WTA circuits based on flipped voltage followers",Circuits and Systems II: Express Briefs, IEEE Transactions on. Vol.52 , No. 7, 2005, pp. 420-423.

[9] Y. Fang, M.A. Cohen, T.G. Kincaid, "Dynamic Analysis of aGeneral Class of Winner-Take-All Competitive Neural Networks",Neural Networks, IEEE Transactions on, Vol. 21, No. 5, 2010, pp.771-783.

[10] M. Rahman, K.L. Baishnab, F.A. Talukdar, "A high speed and highresolution VLSI Winner-take-all circuit for neural networks andfuzzy systems", Signals, Circuits and Systems, ISSCS. InternationalSymposium on. 2009, pp. 1-4 .

[11] S. Kazemi Nia, A. Khoei, Kh. Hadidi,“ High Speed High PrecisionVoltage-Mode MAX and MIN Circuits," Journal of circuits, systemsand computer (JCSC), Vol. 16, No. 2, PP. 233-244, April 2007.

[12] R.G. Carvajal, A. Ramirez Angulo, J. Martinez Heredia," High-SpeedHigh-Precision Min/Max Circuits in CMOS Technology," IEE Electron.Lett. Vol. 36, NO. 8, PP. 697-699, April 2000.

[13] S. Vlassis and S. Siskos, High speed and high resolution WTA circuit, Proc. 1999 IEEE Int. Symp. Circuits and Systems, ICAS'99, Vol. 2, pp. 224-227.

[14] J. Ramirez-Angulo, G. Ducodray-Acevedo, R. G. Carjaval and A. Lopez-Martin, Low-voltage high-performance voltage-mode and current-mode WTA circuit based on flipped voltage followers, IEEE Trans. Circuits Syst. II, Vol. 52 , July 2005.

[15] R. G. Carvajal, J. Ram'irez-Angulo, A. L'opez-Martin, A. Torralba, J. Gal'an, A. Carlosena and F. Munoz, The flipped voltage follower: A useful cell for low-voltage lowpower circuit design, IEEE Trans. Circuits Syst. I, Vol. 52, July 2005. roman 